\title{
Numerical Simulation and Experimental Analysis of the Magnetic Damping Effect generated by a Moving Magnet
}

\author{
Petr Ferfecki1 ${ }^{1,2}$, Marek Konečný1,2, Michal Molčan ${ }^{1,2}$, Jaroslav Zapomě ${ }^{2,3}$ \\ ${ }^{1}$ IT4Innovations, VSB - Technical University of Ostrava, 17. listopadu 2172/15, 70800 Ostrava-Poruba, Czech \\ Republic. E-mail: petr.ferfecki@vsb.cz \\ ${ }^{2}$ Department of Applied Mechanics, VSB - Technical University of Ostrava, 17. listopadu 2172/15, 70800 Os- \\ trava-Poruba, Czech Republic. E-mail: michal.molcan@vsb.cz, marek.konecny@vsb.cz \\ 3Institute of Thermomechanics, The Czech Academy of Sciences, Dolejškova 1402/5, 18200 Praha 8, Czech \\ Republic. E-mail: jaroslav.zapomel@vsb.cz
}

Nowadays, to reduce vibrations of machines, damping devices utilize the eddy current damping effect being increasingly investigated for its advantages of no mechanical contact, no viscous liquid required, high reliability, and good damping capacity. This article studied the main principle of the eddy current damping effect for a moving permanent magnet in a stationary and electrically conductive nonmagnetic cylindrical tube. The magnetic damping coefficient is investigated experimentally, analytically, and by numerical simulations in a steady-state. The numerical simulation is performed in the ANSYS Maxwell programme. The obtained results indicate that the damping force affecting the moving magnet has a viscous form. The experimentally measured and computed results are in good agreement. The effect of varying tube diameter and the tube wall thickness on the magnetic damping coefficient is shown. The contribution of this article consists in the development and a comparison of the obtained results of three approaches for determining the magnetic damping coefficient for a moving magnet in a cylindrical tube.

Keywords: Eddy Current Induction, Permanent Magnet, Dissipative Power, Magnetic Damping Force

\section{Introduction}

In recent years, to suppress the mechanical vibrations of damping devices using smart materials [1], the properties of which can be significantly changed by external electric and magnetic fields, are under development.

Smart materials such as electrorheological [2] or magnetorheological [3] fluids, it has been used in many applications and it can be divided into categories according to the relative direction of the fluid flow and the external magnetic field [4]. A mathematical model of a short magnetorheological damper for a rotordynamic application working in the squeeze mode is developed in $[5,6]$.

A different working principle of damping devices is based on the electromagnetic force due to induction of eddy currents. When a conductor moves in a stationary magnetic field, the eddy currents are induced and Lorenz force is produced [7]. In technical practice, the eddy current principle is also used for non-destructive testing [8]. However, for the transformer or reluctance damper type [9], its generation is achieved by changing reluctance of the magnetic circuit.

The damping characteristics of Lorenz force induced by the eddy currents of a moving permanent magnet in a stationary and electrically conductive non- magnetic cylindrical tube are the principal contribution of this article. The analytical model and the computational model based on the finite element method have been developed, and their validity has been verified by experiment measurement for a copper tube.

\section{Motion Equation of the Permanent Mag- net and the Magnetic Damping Force}

In this work, a permanent magnet falling through a stationary electrically conductive nonmagnetic cylindrical tube is studied. The task was to investigate the kinematic parameters of the falling magnet and the damping coefficient of the eddy current effect utilizing numerical simulations and experimental measurements.

The moving permanent magnetic field generates circulation of the electrical currents within the electrically conductive parts. These eddy currents produce the damping force which arises from the interaction of the two magnetic fields. Since the damping force is proportional to the magnet velocity [10], the magnetic damping has a viscous form.

Current density $\mathbf{J}$ of the induced eddy currents [11] is defined by

$$
\mathbf{J}=\boldsymbol{\sigma}(\mathbf{E}+\mathbf{v} \times \mathbf{B}),
$$


where $\mathbf{E}$ is the electric field intensity, $\mathbf{B}$ is the magnetic flux density of the magnetic field, $\mathbf{v}$ is the velocity of the falling magnet, and $\sigma$ is the electrical conductivity of the tube. Magnetic damping force $\mathbf{F}_{d}$ can be computed as volume $V$ integral [11] from the component of the magnetic field in the direction of motion of the magnet

$$
\mathbf{F}_{d}=\int_{V}(\mathrm{~J} \times \mathbf{B}) \mathbf{d} \boldsymbol{V} .
$$

The damping force direction is opposite to the velocity and it is given by the cross products in Eqs. (1) and (2). The component of magnetic damping force $F_{d z}$ in the $z$ direction due to the induced eddy currents is according to $[10,12]$ defined as linear viscous damping characteristic

$$
F_{d z}=b_{M} v
$$

$v$ is the velocity component in the $z$ direction, $b_{M}$ is the magnetic damping coefficient and it is defined by a relation

$$
b_{M}=\frac{45}{1024}\left(\mu_{0} m\right)^{2} \sigma \frac{R_{0}}{3 R_{0}^{4}}\left(1-\frac{R_{0}^{3}}{R_{1}^{3}}\right),
$$

where $\mu_{0}$ is the vacuum permeability $\left(4 \pi \cdot 10^{-7} \mathrm{H} \cdot \mathrm{m}^{-1}\right)$, $R_{0}$ and $R_{1}$ is the tube inner and outer radius, respectively, and $m$ is the magnetic dipole moment of the magnet

$$
\boldsymbol{m}=\frac{2 B V_{M}}{\mu_{0}} \frac{\sqrt{L_{M}^{2}+R_{M}^{2}}}{L_{M}} .
$$

$V_{M}, L_{M}$, and $R_{M}$ are the volume, height, and radius of the magnet, respectively, and $B$ is the magnetic flux density in the centre of the pole surface.

For the assumption that the tube thickness $\left(R_{1}-\right.$ $R_{0}$ ) is much smaller than the tube inner radius $R_{0}$ is the magnetic damping coefficient defined by

$$
b_{M}=\frac{45}{1024}\left(\mu_{0} m\right)^{2} \sigma \frac{R_{1}-R_{0}}{R_{0}^{4}} .
$$

The motion equation of the falling magnet in the vertical direction $z$ along the stationary cylindrical tube is given by the second Newton law

$$
\boldsymbol{m}_{M} \frac{\mathrm{d} v}{\mathrm{~d} t}+\boldsymbol{b}_{E} v=\boldsymbol{m}_{M} \boldsymbol{g}-\boldsymbol{F}_{d z}
$$

where $m_{M}$ is the mass of the magnet, $b_{E}$ is the external damping coefficient, $g$ is the gravitational acceleration, and $t$ is time.

Varying speed $v(t)$ and vertical position $z(t)$ of the falling magnet inside the tube for initial conditions $v(t=0 s)=0, z(t=0 s)=0$ are derived by the Eq. (7)

$$
\begin{gathered}
v(t)=g \tau\left(1-e^{-t / \tau}\right), \\
z(t)=-g \tau^{2}\left(1-e^{-t / \tau}\right)+g \tau t
\end{gathered}
$$

where time constant $\boldsymbol{\tau}$ is

$$
\tau=\frac{m_{M}}{b_{E}+b_{M}} .
$$

\section{Experimental Measurement and Numeri- cal Simulation of the Eddy Currents In- duction in a Cylindrical Tube}

A set of experiments of the falling magnet inside a cylindrical tube was made for the validation of the computational models for estimation of the eddy current damping. The copper tube and neodymium magnet used for the experiment is shown in Fig. 1.

The material of the magnet is neodymium $\mathrm{NdFeB}$ and its parameters are summarized in Table 1 . The magnetization direction is along the axis of the magnet and the magnetic flux density was measured in the centre of the magnet surface pole with Tesla Meter WT10A.

The geometrical and material parameters of the copper tube are stated in Table 2.

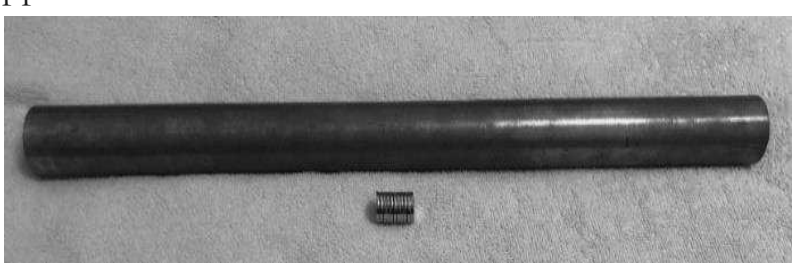

Fig. 1 The tested magnets and the cylindrical tube

In initial position the bottom pole of the magnet was placed near the top surface of the tube and the time measurement was started and it was finished when the bottom pole of the magnet was observed near the end of the tube. The average magnet fall time is $16.26 \mathrm{~s}$ which was computed from twenty-five measurements.

Tab. 1 Geometrical and material parameters of the magnet

\begin{tabular}{lll}
\hline Parameter Name & Value & Units \\
\hline Diameter & 15 & $\mathrm{~mm}$ \\
Height & 20 & $\mathrm{~mm}$ \\
Magnet mass & 0.026 & $\mathrm{~kg}$ \\
Electrical conductivity & $6.25 \cdot 10^{5}$ & $\mathrm{Sm}^{-1}$ \\
Magnetic flux density & 0.485 & $\mathrm{~T}$ \\
Relative permeability & 1.0446 & - \\
\hline
\end{tabular}

Tab. 2 Geometrical and material parameters of the copper tube

\begin{tabular}{lll}
\hline Parameter Name & Value & Units \\
\hline Outer diameter & 36 & $\mathrm{~mm}$ \\
Inner diameter & 18 & $\mathrm{~mm}$ \\
Tube length & 450 & $\mathrm{~mm}$ \\
Electrical conductivity, from [10] & $3.58 \cdot 10^{7}$ & $\mathrm{Sm}^{-1}$ \\
Relative permeability & 1 & - \\
\hline
\end{tabular}


The relation for the magnetic damping coefficient derived in this paper is based on the following assumptions: (i) the damping force of the induced eddy currents is directly proportional to the velocity, (ii) the motion of the falling magnet is uniform, and (iii) the viscous coefficient of the external damping and the friction damping between the magnet and the tube wall are negligible. The inclusion of an intermittent impact between the magnet and the tube is presented in article [13], where one can find the relevant solver parameters settings.

Inserting a relation for the velocity of uniform motion into the steady-state solution of the motion equation Eq. (7) leads to the expression of the magnetic damping coefficient

$$
b_{M}=\frac{m_{M} g t_{F}}{L},
$$

where $L$ is the tube length, and $t_{F}$ is the magnet fall time. The steady-state damping coefficient from an experiment determined by Eq. (11) is $9.22 \mathrm{~kg} \cdot \mathrm{s}^{-1}$.

The ANSYS Maxwell program was used for solving motion equation Eq. (7) of the falling magnet. A $2 \mathrm{D}$ axial symmetric problem of the magnet in the cylindrical tube has to be solved. In the simulation it is assumed that the magnet is rigid, the eddy currents are induced only in the cylindrical tube, and the external damping is negligible.

Around the permanent magnet and the cylindrical tube an air was modelled. A zero value of the vector potential of the magnetic field was imposed on the air outer border. The initial velocity of the magnet was zero and its position was the same as the tube edge.

The domain was discretized by 180015 of the quadratic triangular elements. The translational motion was solved by the time integration and it was carried out on a workstation (CPU: Intel Xeon E5-2640 v3, RAM Memory: $256 \mathrm{~GB}$ ) and the solution time was about $300000 \mathrm{~s}$.

In Fig. 2, the magnitude of the magnetic flux density in the magnet and tube at the uniform magnet motion is depicted. The results show that the maximum value of the flux density is reached on the outer cylindrical surface and in the middle length of the magnet. It is clear that the eddy currents are induced mainly near the surface of the inner diameter tube and close to the magnet poles.

Time history of the magnet displacement and velocity in the vertical direction is drawn in Fig. 3 and Fig. 4. The analytical solution of the motion equation Eq. (7) with the magnetic damping coefficient determined by the experiment is depicted in Figs. 3 and 4 with the red dashed line. The solution is displayed in the instantaneous times that correspond to the initial and end position (are marked with black crosses in Figs. 3 and 4) of the magnet located under and above the edge of the tube. The initial magnet velocity sharply increased and decreased, and the subsequent motion is almost uniform and as the magnet approached the tube edge, its velocity increased again. The uniform velocity is computed from the measured tube length and the time to reach the tube end since the transient time is short. The mean values of the uniform velocity determined from the numerical simulation and the analytical-experimental solution equal to $28.32 \mathrm{~mm} \cdot \mathrm{s}^{-1}$ and $27.68 \mathrm{~mm} \cdot \mathrm{s}^{-1}$, respectively. It may be seen that the agreement between the numerical simulation and experiment is very good.
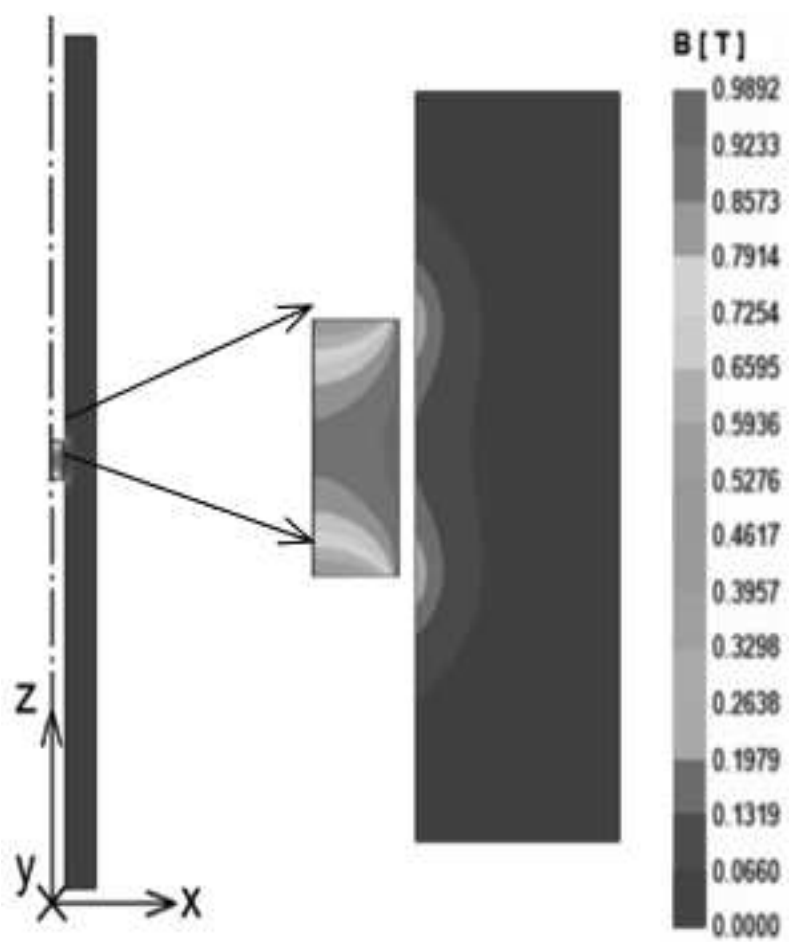

Fig. 2 Distribution of the magnetic flux density in the magnet and tube and a detail view

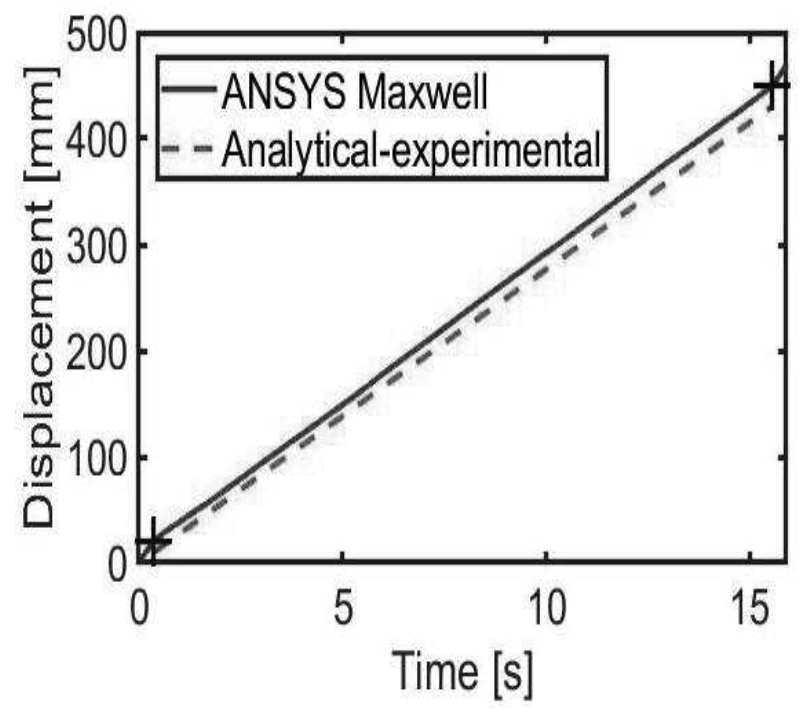

Fig. 3 Time history of the displacement 


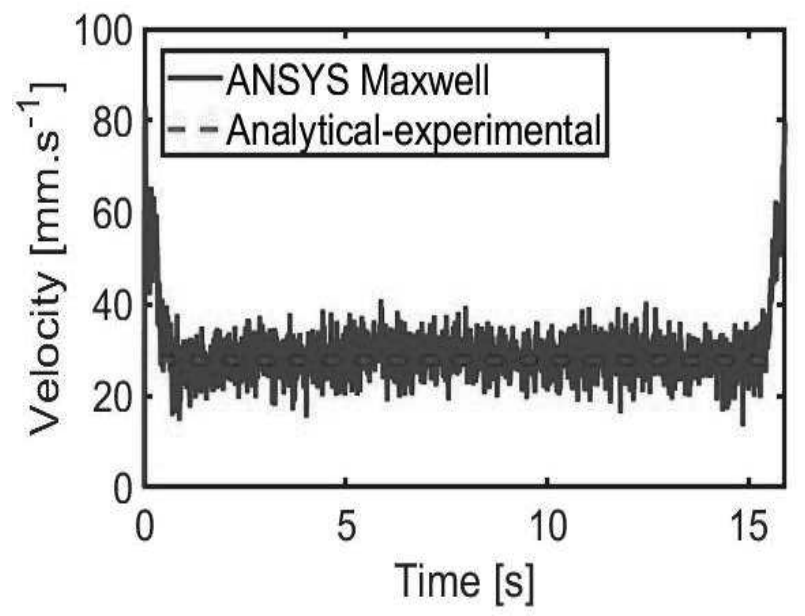

Fig. 4 Time history of the magnet velocity

In Fig. 5 and 6 the time dependences of the damping force and the dissipative power are depicted. The mean values of the damping force and the dissipative power during the uniform motion of the magnet equal to $260 \mathrm{mN}$ and $7 \mathrm{~mW}$, respectively. The variations in the time history of the velocity, damping force, and dissipative power are caused by numerical reasons such as a moving mesh, size of a time step, and a mesh quality.

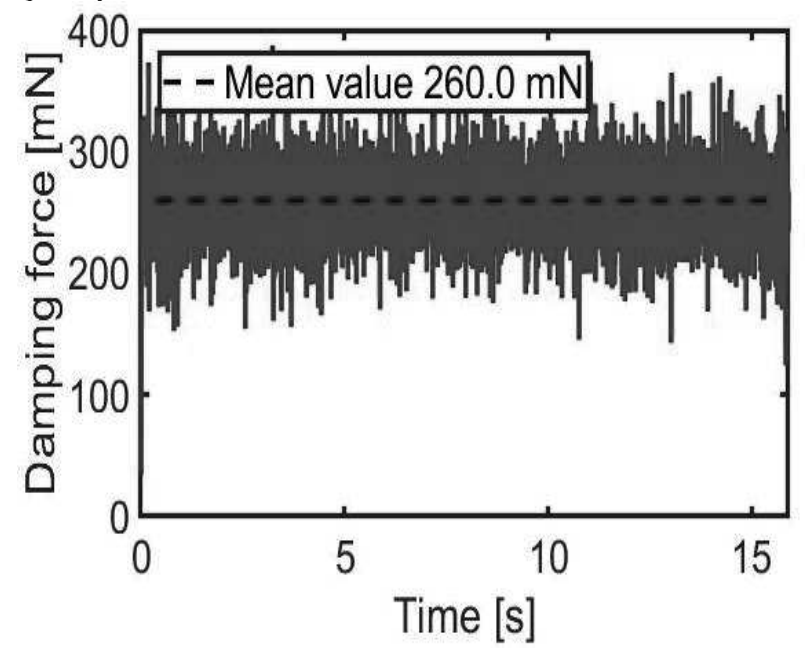

Fig. 5 Time history of the damping force

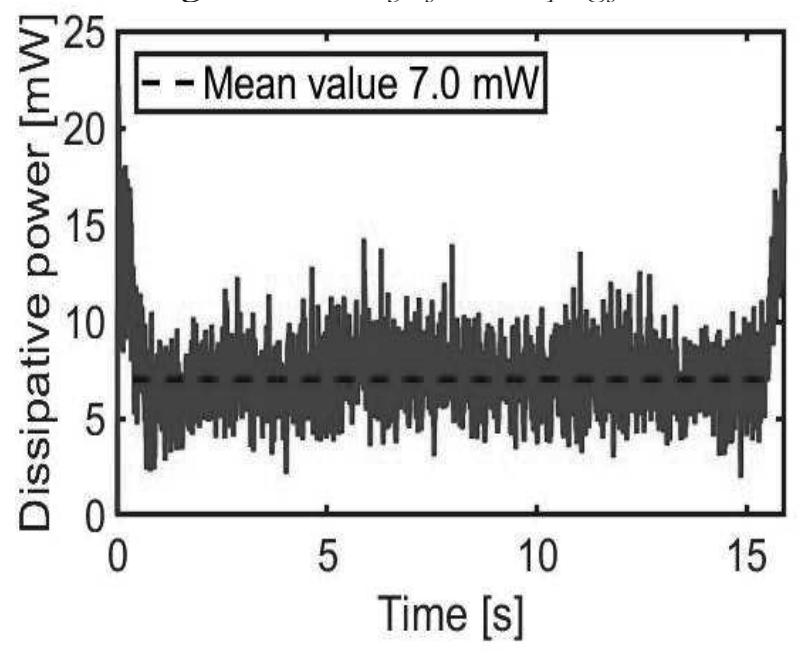

Fig. 6 Time history of the dissipative power
Tab. 3 The magnetic damping coefficient

\begin{tabular}{lll}
\hline Type & Value & Units \\
\hline Experiment & 9.22 & $\mathrm{~kg} \cdot \mathrm{s}^{-1}$ \\
Numerical simulation & 9.18 & $\mathrm{~kg} \cdot \mathrm{s}^{-1}$ \\
Analytical solution & 8.44 & $\mathrm{~kg} \cdot \mathrm{s}^{-1}$ \\
\hline
\end{tabular}

The magnetic damping coefficient determined by means of the experiment and by two computational models is given in Table 3. The damping coefficient for the experiment and the analytical solution is determined by Eqs. (11) and (4), respectively. For results of the uniform motion of the magnet obtained by the ANSYS Maxwell program is the damping coefficient computed by Eq. (3). The achieved results show a very good agreement, see Table 3.

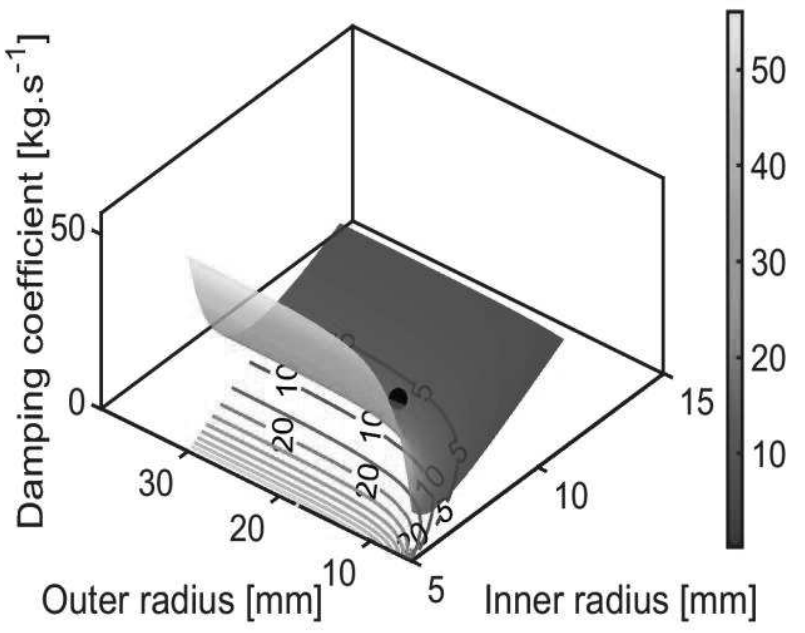

Fig. 7 Dependency of the magnetic damping coefficient on the tube outer and inner radius and for a constant volume of the magnetic dipole moment and the black point indicates the parameters of the tested tube

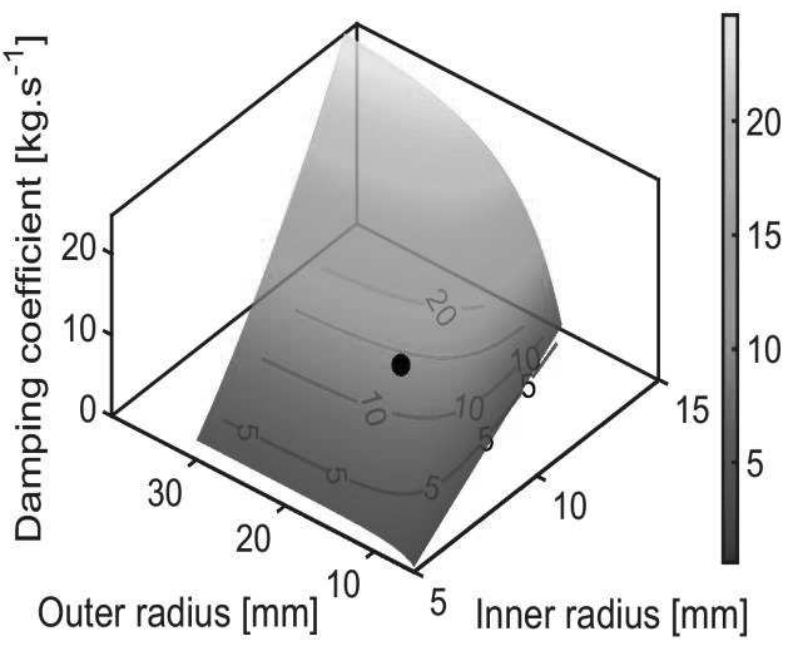

Fig. $\boldsymbol{8}$ Dependency of the magnetic damping coefficient on the tube outer and inner radius and for a variable volume of the magnetic dipole moment and the black point indicates the parameters of the tested tube 
The magnetic damping coefficient in dependence on the tube outer and inner radius is depicted in Fig. 7 and 8 . The damping coefficient was computed for the assumption that the magnetic flux density is a constant. In Fig. 7 the magnitude of the magnetic dipole moment of the magnet is independent of the tube inner radius and in Fig. 8 its volume is dependent on the tube inner radius.

In Fig. 9 and 10 dependency of the magnetic damping coefficient on the tube inner radius for four tube thickness is drawn. The damping coefficient was computed for assumptions that the magnetic flux density has a constant value and the tube thickness is much smaller than the tube inner radius (Eq. 6). In Fig. 9 the magnitude of the magnetic dipole moment of the magnet is independent of the tube inner radius. The damping coefficient increases as the tube inner radius decreases and its thickness increases. If the magnetic dipole moment is dependent on the tube inner radius (Fig. 10), the damping coefficient and the tube inner radius are increasing.

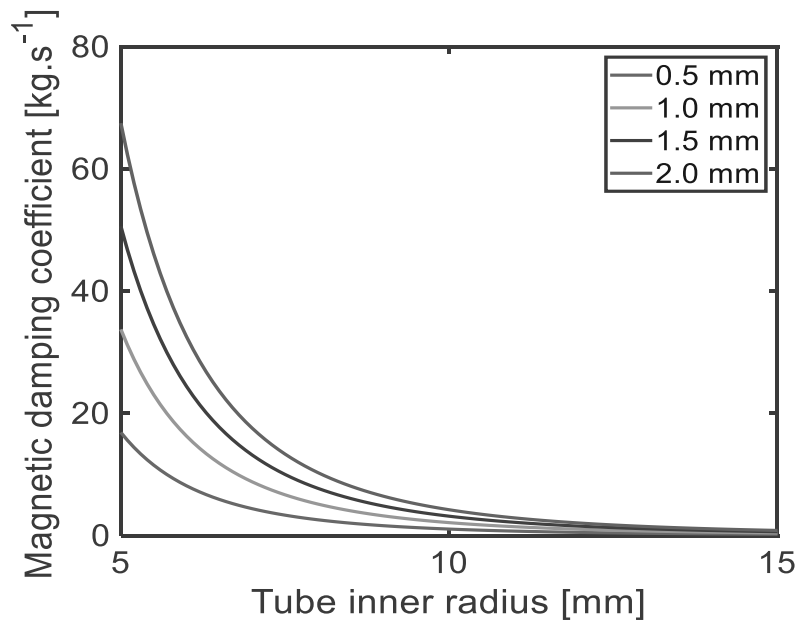

Fig. 9 Dependency of the magnetic damping coefficient on the tube inner radius for the four tube thicknesses and a constant volume of the magnetic dipole moment.

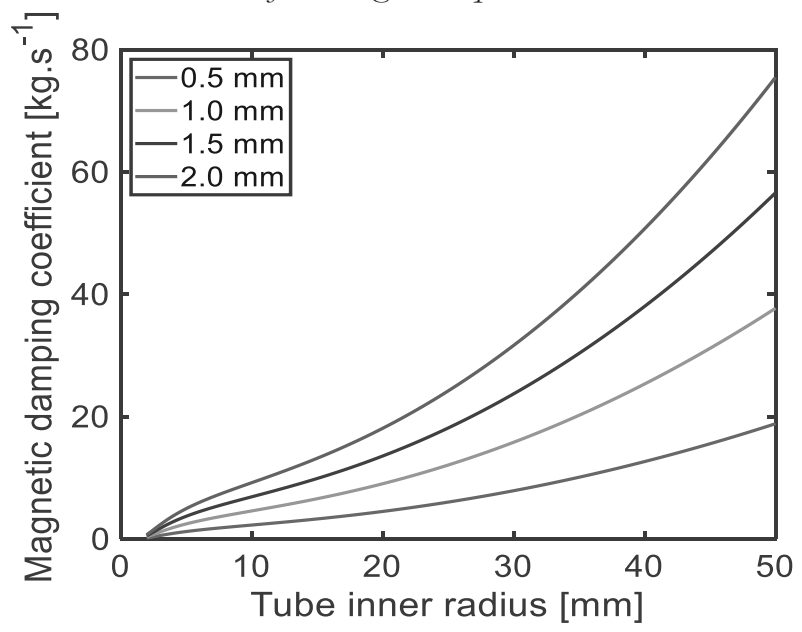

Fig. 10 Dependency of the magnetic damping coefficient on the tube inner radius for the four tube thicknesses and a variable volume of the magnetic dipole moment.

\section{Conclusions}

The numerical and analytical computational model to explain the motion of the permanent magnet inside a vertical conductive tube has been presented. The effect of varying the tube inner and outer radius was studied. The used computational models were experimentally validated. The small discrepancies between the experimental measurement and the computational results were read. The relative error between the experiment and the numerical, or analytical result of the magnetic damping coefficient is about $0.5 \%$ and $8.5 \%$, respectively. The findings achieved in this work will be used to design a damping device for attenuation of the bending vibration of the rotor system.

\section{Acknowledgments}

This work was supported by The Ministry of Education, Youth and Sports from the National Programme of Sustainability (NPU II) project „IT4Innovations excellence in science - LQ1602“, by the grant project 19-06666S of the Czech Science Foundation, and by the "Student's Grant Competition project SP2020/139“.

\section{References}

[1] HAJALILOU, A., MAZLAN, S. A., LAVVAFI, H., SHAMELI, K. (2016). Field Responsive Fluids as Smart Materials. 1st edn. Springer, Singapore (2016).

[2] SHENG, P., WEN, W. (2012). Electrorheological Fluids: Mechanisms, Dynamics, and Microfluidics Applications. Annual Review of Fluid Mechanics 44, 143-174 (2012).

[3] VICENTE, J., KLINGENBERG, D. J., HIDALGO-ALVAREZA, R. (2011). Magnetorheological fluids: a review. Soft Matter 7, 3701-3710 (2011).

[4] GONG, X., RUAN, X., XUAN, S., YAN, Q., DENG, H. (2015). Magnetorheological Damper Working in Squeeze Mode. Advances in $\mathrm{Me}$ chanical Engineering 6, 1-10 (2015).

[5] ZAPOMĚL, J., FERFECKI, P. (2018). The Oscillation Attenuation of an Accelerating Jeffcott Rotor Damped by Magnetorheological Dampers Affected by the Delayed Yielding Phenomenon in the Lubricating Oil. Journal of Vibration and Acoustics 140(1), 1-10 (2018).

[6] FERFECKI, P., ZAPOMĚL, J., MAREK, M. (2017). Multi-physical analysis of the forces in the flexible rotor supported by the magnetorheological squeeze film dampers. 12th International Conference on the Theory of Machines and 
Mechanisms 2016, vol. 44. Mechanisms and Machine Science (2017).

[7] ZUO, L., CHEN, X., NAYFEH, S. (2017). Design and Analysis of a New Type of Electromagnetic Damper With Increased Energy Density. Journal of Vibration and Acoustics 133(4), 1-8 (2017).

[8] BENEŠ, P., PRŮCHA, V., HÁJEK, J., VÍTOVEC, V. (2019). Possibility of Using Eddy Current Evaluation of the Heat Treatment of Steel. Manufacturing Technology 19(3), 371-374 (2019).

[9] AmATI, N., SILVAGNI, M., TONOLI, A. (2008). Transformer Eddy Current Dampers for the Vibration control. Journal of Dynamic Systems, Measurement and Control 130(9), 1-9 (2008).

[10] DONOSO, G., LADERA, C. L., MARTIN, P. (2009). Magnet fall inside a conductive pipe: motion and the role of the pipe wall thickness. European Journal of Physics 30(4), 855-869 (2009).

[11] KNOEPFEL, H. E. (2000). Magnetic Fields: Comprehensive Theoretical Treatise for Practical Use. 1 st edn. John Wiley \& Sons, Inc. New York (2000).

[12] UHLIG, R. P., ZEC, M., BRAUER, H. (2012). Lorentz Force Eddy Current Testing: a Prototype Model. Journal of Nondestructive Evaluation, Journal of Nondestructive Evaluation 31, 357-372 (2012).

[13] ŠULKA, P., SAPIETOVÁ, A., DEKÝŠ, V., SAPIETA, M. (2018). Analysis and synthesis parameters influencing to the effects of impact. In: Vasko, M., et al. (eds.) XXII Slovak-Polish Scientific Conference on Machine Modelling and Simulations 2017, vol. 157. MATEC Web of Conferences (2018). 